Methods: A total of 74 patients, diagnosed according to the modified New York criteria for AS, were enrolled. Patients were assessed to measure disease activity using the Bath Ankylosing Spondylitis Disease Activity Index [BASDAI]. Fear of movement was assessed with the Tampa Scale for Kinesiophobia [TSK]. To calculate NSAID intake and the type of NSAID, dose, percentage of days with intake were recorded, along with DMARD therapy, age, body mass index (BMI), and disease duration. The NSAID equivalent scoring was calculated according to recommendations from longitudinal clinical studies. The drug therapy groups were compared using the Kruskal-Wallis test and the Chi-square test. Correlation analysis was evaluated by Spearman's correlation coefficient.

Results: Seventy-four patients (36 women, 38 men; mean age: $43.81 \pm 10.18$ years; mean disease duration: $9.89 \pm 8.50$ years; $\mathrm{BMI}$ : $28.20 \pm 5.07)$ treated with four types of DMARDs (adalimumab+golimumab $=17$; infliximab $=19$; etanercept $=13$; sulfasalazine $=25$ ) were included. There were no drug group differences in terms of age $(p=0.179)$, sex $(p=0.886)$, or BMI $(p=0.821)$. BASDAI scores (mean: $3.9 \pm 2.4$ ) and NSAID intake (mean: $68.1 \pm 76.1 ; p=0.003$ ) were significantly higher in the sulfasalazine therapy (ST) group compared to other drug groups. BASDAI scores were not correlated with age $(p=0.103)$, disease duration $(p=0.131)$, BMI $(p=0.641)$ or the TSK scores $(p=0.376)$. Different NSAID intake groups $(p=0.089)$ had similar TSK scores.

Conclusions: Patients with AS had fear of movement independent of age, BMI or disease duration, even when they experienced positive results from drug therapies and concomitant therapy with a single oral dose of NSAID or oral corticosteroids in stable dosages.

References:

[1] Braun J, Baraliakos X, Heldmann F, et al. Tumor necrosis factor alpha antagonists in the treatment of axial spondyloarthritis. Expert Opin Investig Drugs. 2014;23:647-659.

[2] Rohekar S, Chan J, Tse SM, et al. 2014 update of the Canadian Rheumatology Association/Spondyloarthritis Research Consortium of Canada treatment recommendations for the management of spondy- loarthritis. Part I: principles of the management of spondyloarthritis in Canada. J Rheumatol. 2015;42:654664

[3] Rohekar S, Chan J, Tse SM, et al. 2014 update of the Canadian Rheumatology Association/Spondyloarthritis Research Consortium of Canada treatment recommendations for the management of spondy- loarthritis. Part II: specific management recommendations. J Rheu- matol. 2015;42:665-681.

Acknowledgements: We would like to thank Rheumatology Nurse Ayten Yuksek in our department for monitoring and documenting of the data, and our patients for assistance with their valuable participation to our study.

Disclosure of Interest: None declared

DOI: 10.1136/annrheumdis-2017-eular.1389

\section{SAT0757-HPR SIGNIFICANT IMPROVEMENT OF RHEUMATOID ARTHRITIS (RA) OUTCOME WITH REPEATED SELF-ASSESSMENT APPLYING SMART SYSTEM OF DISEASE MANAGEMENT (SSDM) MOBILES TOOLS: A COHORT STUDY OF RA PATIENTS IN CHINA}

J. Yang ${ }^{1}$, Y. Wang ${ }^{2},{\mathrm{~F} . \mathrm{Li}^{3}}^{3}, \mathrm{H}$. Wei ${ }^{4}, \mathrm{~J} \mathrm{Xue}{ }^{5}, \mathrm{H} . \mathrm{Wang}^{6}$, J. Ru ${ }^{7}, \mathrm{R} . \mathrm{Mu}^{8}$, J. Huang ${ }^{9}$, X. Zuo ${ }^{10}$, X. Xin ${ }^{11}$, F. He ${ }^{12}$, X. Shi ${ }^{13}$, Z. $\mathrm{Li}^{14}$, F. Xiao ${ }^{15}, \mathrm{H}$. Xiao $^{15}$, Y. Liu ${ }^{15}$, Y. Jia ${ }^{15}$, Z. $\mathrm{Li}^{8} .{ }^{1}$ Department of Rheumatology, Central Hospital of MianYang, Sichuan, Mianyang; ${ }^{2}$ Department of Rheumatology, The First Affiliated Hospital of BaoTou Medical College, Baotou; ${ }^{3}$ Department of Rheumatology and Immunology, The Second Xiangya Hospital of Central South University, Changsha; ${ }^{4}$ Department of Rheumatology, Northern Jiangsu People's Hospital, Yangzhou; ${ }^{5}$ Department of Rheumatology and Immunology, The Second Affiliated Hospital of Zhejiang University School of Medicine, Hangzhou; ${ }^{6}$ Department of Rheumatology and Immunology, JiaXing First Hospital, Zhejiang, Jiaxing; ${ }^{7}$ Dept. of Rheumatology, The 264th Hospital of the PLA, Taiyuan; ${ }^{8}$ Department of Rheumatology and Immunology, People's Hospital, Beijing University Medical School, Beijing; ${ }^{9}$ Department of Rheumatology and Immunology, The sixth Affiliated Hospital of Sun Yat-sen University, Guangzhou; ${ }^{10}$ Department of Rheumatology and Immunology, Xiangya Hospital Centeral South University, Changsha; ${ }^{11}$ Ningbo First Hospital, Zhejiang, Ningbo;

${ }^{12}$ Department of Rheumatology, Suining Central Hospital, Suining; ${ }^{13}$ Department of Rheumatology and Immunology, The First Affiliated Hospital of Henan University of Science and Technology, Luoyang; ${ }^{14}$ Department of rheumatology, The First Affiliated Hospital of Bengbu Medical College, Bengbu; ${ }^{15}$ Medical Department, Shanghai Gothic Internet Technology Co., Ltd., Shanghai, China

Background: There are more than 5 million RA patients in China, but only 5,000 rheumatologists. Treat-to-Target (T2T) strategy are critical for the treatment of $\mathrm{RA}$, but the Chinese rheumatologists can hardly provide patients with a complete assessment in the clinic due to limited time. The SSDM includes interfaces of both physicians' and patients' application. After entering the data of lab test records, treatment regiments, and executing DAS28 assessment by patients themselves, all data can be synchronized automatically to the authorized physicians' mobile tool. The rheumatologists can adjust treatment regiments base on patients' profile. Our previous study showed that patients in China can master the application of SSDM for accurately evaluating DAS28 and health assessment questionnaire (HAQ) after training.

Objectives: The purpose of this study is to explore the effectiveness of applying
SSDM in improvement of disease activity after repeated self-assessment in Chinese RA patients.

Methods: Patients were educated to assess DAS28 with SSDM and asked to repeat the self-assessment once a month. Descriptive statistics were performed for patient and disease characteristics. According to DAS28 scores, disease activity was divided into four groups: remission (Rem), low disease activity (LDA), moderate disease activity (MDA) and high disease activity (HDA).

Results: From Aug 2014 to Jan 2017, a total of 11,867 RA patients from 459 centers in China participated in the study. The mean age was 49.27 \pm 14.35 (18 to 99 ) years and the median disease duration was 11.43 months. All patients performed self-assessment of DAS28, HAQ and morning stiffness time for 20,376 times. Proportion of patients in Rem, LDA, MDA and HDA was 16\%, 12\%, 46\% and $25 \%$ respectively at baseline. Of which, 3,472 patients performed repeated assessment for 8,509 times. Proportion of patients in Rem, LDA, MDA and HDA changed into $34 \%, 18 \%, 38 \%$ and $10 \%$ at the last assessment. The proportion of T2T (DAS28 <3.2) at the last assessment was higher than that baseline significantly $(P<0.001)$. According to the assessments, the rate of T2T from baseline to 6 times were $28 \%, 41 \%, 49 \%, 51 \%, 56 \%$ and $58 \%$ (see Figure 1).With the increase of the times of self-assessment, the T2T rate was significantly improved $(p<0.001)$.

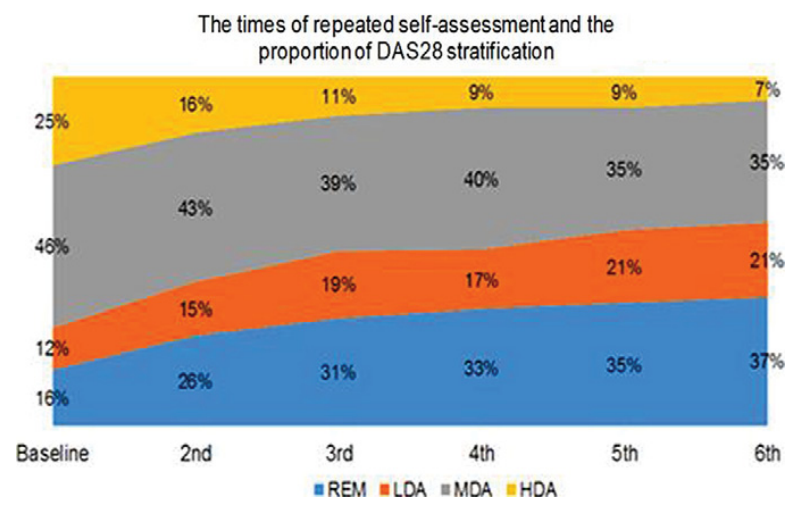

Conclusions: Under repeated self-assessment of DAS28 using SSDM, RA patients can achieve better T2T result. SSDM can assist rheumatologist to rationally adjust treatment for RA patients.

Disclosure of Interest: None declared

DOI: 10.1136/annrheumdis-2017-eular.5042

\section{SAT0758-HPR DEPRESSION BUT NOT CORE SYMPTOMS PREDICT CAREGIVER STRAIN IN ADULTS SHARING A HOME WITH PRIMARY SJÖGREN'S SYNDROME PATIENTS}

K. Hackett $^{1}$, D. Lendrem ${ }^{1}$, T. Rapley ${ }^{1}$, V. Deary ${ }^{2}$, K. Deane ${ }^{3}$, J. Newton ${ }^{1}$, W.-F. Ng ${ }^{1}$. ${ }^{1}$ Newcastle University; ${ }^{2}$ Northumbria University, Newcastle Upon Tyne; ${ }^{3}$ University of East Anglia, Norwich, United Kingdom

Background: Patients with primary Sjögren's syndrome (PSS) experience a range of symptoms including dryness, fatigue, pain and low mood. The impact of these symptoms upon the people with whom they live, is unknown. The caregiver strain index (CSI) is a thirteen item questionnaire which measures strain in caregivers (Robinson 1983).

Objectives: To determine whether adults living in the same household as a person with PSS experience caregiver strain and to examine the relationship between caregiver strain and other factors.

Methods: We collected age, Carer Strain Index (CSI) and quality of life (QOL) (SF-36) scores from 62 adults who shared a home with a PSS patient. We also collected various measures from the PSS patients that they lived with. The patient measures included; age, time since diagnosis, fatigue, dryness, pain, $\mathrm{QOL}$, depression, anxiety, and physical function (Improved HAQ). We conducted Spearman correlations to compare the relationship between the carers' CSI and SF-36 scores as well as the measures from the patients they lived with. We performed multivariate analyses on factors that significantly correlated with CSI scores.

Results: The caregiver strain scores are shown in the Figure. Although a median CSI score of 1 demonstrates that most AHM participants experienced very little caregiver strain, $14 \%$ of $\mathrm{AHM}$ participants did experience high levels (indicated with a CSI score $\geq 7$ )

Age and PSS disease duration negatively correlated with carer strain. Increased pain, fatigue, mental fatigue, physical functioning, anxiety, depression in the patients and SF-36 physical component scores in the household members all significantly correlated with increased carer strain. There was no relationship between dryness scores and age of the PSS patient and CSI scores.

Multivariate analysis suggests the main predictor of increased carer strain was depression in the PSS patient and younger age of the household member

Conclusions: Caregiver strain is evident in a proportion of adults sharing a home with a person with PSS. Caregivers of patients with high depression scores are particularly susceptible and may benefit from additional support. 